\title{
ABSTRACTS OF CONTRIBUTED PAPERS
}

\section{OSCILLATING STARS (MIRAS), MASS LOSS AND FORMATION OF PLANETARY NEBULAE (PN)}

I. Tuchman, N. Sack, and Z. Barkat

The Hebrew University of Jerusalem, Israel

We report some results of an extensive study of dynamically and/or pulsationally unstable stellar envelopes at the red giant stage.

Both a static survey and numerical hydrodynamical calculations have been performed. Earlier suggestions (Smith and Rose 1972; Wood 1974) for a mechanism which gives rise to mass ejection and PN formation (via repeated shocks), are substantiated and expanded.

New results concerning the qualities of Mira variables and their association with PN are obtained and discussed.

ARE LIGHT DA WHITE DWARFS THE PROGENITORS OF PLANETARIES?

\author{
Arrigo Finzi \\ Israel Institute of Technology, Haifa, Israel
}

According to a model presented elsewhere the progenitors of planetaries are fully degenerate stars with hydrogen-rich outer shells and internal temperatures lower than those of observed white dwarfs. Apparently, these stars have gone through the white dwarf stage and evolved into invisible black dwarfs. This suggests that some among observed white dwarfs may be stars with hydrogen-rich envelopes that have still to go through the planetary stage. Tentatively, we identify these stars with light white dwarfs of DA spectra and consider possible tests of this tentative identification: 
1. The raddii of light white dwarfs with DA spectra should be larger than those predicted by the mass-radius relation for $\mu_{e}=2$.

2. Among light white dwarfs, those with DA spectra should be comparatively fast rotators.

3. White dwarfs in young stellar systems should have DA spectra.

The nuclear deflagrations that cause the ejection of the shells are possibly ignited by meteorites hitting the black dwarfs.

THE HERBIG-HARO OBJECTS - BREAKUP OF PROTOSTELLAR COCOONS

M.A. Dopita

Mount Stromlo and Siding Spring Observatory, Australia

From a large body of data gathered on the 3.9 metre AAT and the 2 metre and $76 \mathrm{~cm}$ telescopes at Mount Stromlo including absolute photometry, quantitative spectrometry and studies of internal velocity fields, the Herbig-Haro objects are shown to be radiating shock fronts in the ISM. Physical conditions are found by comparison with theoretical models of plane-parallel shock fronts. Shock velocities in the range $30-150 \mathrm{~km} / \mathrm{sec}$ are observed, and the densities in the interstellar clouds in which the objects are embedded found to be $80-400 \mathrm{~cm}^{-3}$. Extinction at $\mathrm{H}_{\beta}$ ranges up to $4.4 \mathrm{mag}$. Parameters are found to be consistent with the radiation pressure driven breakup of a dust cocoon around a newly formed B-type star. 